\title{
Variable-angle epifluorescence microscopy characterizes protein dynamics in the vicinity of plasma membrane in plant cells
}

\author{
Tong Chen ${ }^{1 \dagger}$, Dongchao $\mathrm{Ji}^{1,2+}$ and Shiping $\operatorname{Tian}^{1,2,3^{*}}$
}

\begin{abstract}
Background: The assembly of protein complexes and compositional lipid patterning act together to endow cells with the plasticity required to maintain compositional heterogeneity with respect to individual proteins. Hence, the applications for imaging protein localization and dynamics require high accuracy, particularly at high spatio-temporal level.

Results: We provided experimental data for the applications of Variable-Angle Epifluorescence Microscopy (VAEM) in dissecting protein dynamics in plant cells. The VAEM-based co-localization analysis took penetration depth and incident angle into consideration. Besides direct overlap of dual-color fluorescence signals, the co-localization analysis was carried out quantitatively in combination with the methodology for calculating puncta distance and protein proximity index. Besides, simultaneous VAEM tracking of cytoskeletal dynamics provided more insights into coordinated responses of actin filaments and microtubules. Moreover, lateral motility of membrane proteins was analyzed by calculating diffusion coefficients and kymograph analysis, which represented an alternative method for examining protein motility.
\end{abstract}

Conclusion: The present study presented experimental evidence on illustrating the use of VAEM in tracking and dissecting protein dynamics, dissecting endosomal dynamics, cell structure assembly along with membrane microdomain and protein motility in intact plant cells.

Keywords: Endosomal dynamics, Microdomain, Plasma membrane, Variable-angle epifluorescence microscopy

\section{Background}

Like other cellular membranes, plasma membranes (PMs) are highly organized structures subcompartmented in different functional domains, and they encompass a complex set of proteins and lipids essential for cell morphogenesis and patterning $[1,2]$. The PM provides an environment in which macromolecules interact efficiently, including the clustering of proteins in oligomeric complexes via protein-protein or protein-lipid interactions, the docking and anchoring of protein complexes for regulatory reactions and other precisely orchestrated processes [1]. Furthermore, the coupling of signal

\footnotetext{
* Correspondence: tsp@ibcas.ac.cn

${ }^{\dagger}$ Equal contributors

${ }^{1}$ Key Laboratory of Plant Resources, Institute of Botany, Chinese Academy of Sciences, Nanxincun 20, Xiangshan, Haidian District, Beijing 100093, China

${ }^{2}$ University of Chinese Academy of Sciences, Beijing 100049, China

Full list of author information is available at the end of the article
}

perception with cytoskeletal structures and intracellular second messengers also necessarily involves transduction across the PM. Therefore, the functional dissection of underlying cellular events in the vicinity of the PM, utilizing state-of-the-art techniques, is a priority.

Total internal reflection fluorescence (TIRF), also termed evanescent wave fluorescence, employs evanescent waves to selectively excite fluorophores at the glass / specimen interface and in close proximity to it (100-200 nm) $[3,4]$. As a result of total internal reflection (TIR), the evanescent wave forms and propagates immediately beneath the cell surface, exciting only fluorophores within range of the evanescence field. By contrast, fluorescent particles outside of this range remain unexcited, which makes this technique particularly suitable for dissecting molecular and cellular events in close proximity to the PM [4]. TIRF has been used effectively for imaging protein dynamics in animal cells, such as epidermal growth factor 
dynamics [5], cytoskeletal dynamics [6] and ion channel activity [7]. It depends on the capability of the sample to adhere to the coverslip, however, and to remain within the imaging area defined by the evanescence field.

In plants, however, the plant cell wall imposes a rigid barrier to the real-time tracking of the dynamics of such events with high spatio-temporal accuracy, and this had significantly hampered the efforts of the plant cell research community. Some targets of interest, such as the cells of various plants and fungi, either do not adhere well to coverslips or have thick walls which the evanescence field cannot penetrate well $[8,9]$, which makes the applications of TIFM in plant and fungal cells more complex. However, the penetration depth of TIRF microscopy depends on the incident angle of illumination, resulting in a range of available depths $[3,4]$. Variableangle epifluorescence microscopy (VAEM) allows laser beam to penetrate the cell wall using a sub-critical angle which was smaller than the critical angle [10]. When the beam is refracted at a steep angle, a slanted "sheet" of light is produced. This technique also produces images with a higher contrast than epifluorescence illumination. Without exception, all single-molecule studies on plant cells using TIRF, VAEM or Highly Inclined and Laminated Optical sheet (HiLo) microscopy [11] are conducted based on this principle.

Here, we present experimental evidence showing the breadth of applications of VAEM in the tracking of protein dynamics involved in organellar dynamics, cytoskeletal structure assembly, membrane microdomain organization and protein motility. These applications span right across the plant science community and, besides revealing the underlying mechanisms of processes linked to the PM, they will undoubtedly stimulate new research directions.

\section{Methods \\ Construction of pABP1:ABP1-YFP binary expression vectors}

A genomic fragment of AT4G02980 and the upstreaming sequence of 1500-bp were amplified from genomic DNA samples with primers 5'-CACCAATCTTCATTCTTTAC CTGCAC -3' and 5'- AAGCTCGTCTTTTTGTGAT TCTTG-3', subcloned into pENTR/SD/D-TOPO vectors (Invitrogen), and then sub-cloned into destination vector pMDC107 by LR recombination reaction. Arabidopsis thaliana ecotype Columbia wild-type plants were transformed with the fluorescent-tagged ABP1 constructs using the Agrobacterium tumefaciens-mediated floral dip method [12]. Kanamycin-resistant transgenic plants were grown on solid medium (1\% agar), 1/2 MS medium containing $50 \mu \mathrm{g} / \mathrm{mL}$ kanamycin [13]. 35S:FLOT1a-mCherry is constructed by replacing GFP with mCherry in 35S:FLOT1a-GFP [14].

\section{Plant materials and growth conditions}

Arabidopsis seedlings were maintained as described before [15]. Arabidopsis lines transformed with 35S:FLOT1aGFP [14], pCLC:CLC-GFP [16], 35S:LTi6a-GFP [17], 35S:GFP-fABD2 and 35S:mCherry-TUA5 [18], 35S:BOR1GFP [19], pSKU5:SKU5-GFP/sku5 [20], 35S:StREM-GFP [21], 35S:COBRA-GFP [22], 35S:AtREM1.2-YFP [23], pREM1.2:AtREM1.2-YFP [24] have been described before. Transgenic plants expressing combinations of GFP, mCherry, and YFP fusion proteins were generated by crosspollination, and F1 or F2 generations were used for microscopic observations. Seeds were sterilized with 70\% ethanol, $0.1 \%$ Triton X-100 / 95\% ethanol and plated on half-strength Murashige and Skoog medium $(1 / 2 \mathrm{MS})+1 \%$ agar, stratified for $2-3$ days and grown vertically in the chamber with $7000-10,000 \mathrm{~lx}$ of illuminance for $16 \mathrm{~h}$ per day at $22 \pm 2{ }^{\circ} \mathrm{C}$.

\section{Laser scanning confocal microscopy (LSCM)}

Arabidopsis seedlings were imaged with an Olympus FV1000 MPE Multiphoton Laser Scanning Confocal Microscope (60× water-immersion objective; numerical aperture, 1.35). Both GFP and FM4-64 were excited using a 488-nm laser. The fluorescence emission spectra were separated with a 560LP dichroic mirror. GFP fluorescence was collected in the range of 495 to $540 \mathrm{~nm}$, and that of FM4-64 was collected in the range of 570 to $650 \mathrm{~nm}$. mRFP and mCherry were imaged by a $543-\mathrm{nm}$ laser, and the emission fluorescence of mRFP was collected in the range of 580 to $620 \mathrm{~nm}$ and that of $\mathrm{mCherry}$ in the range of 600 to $650 \mathrm{~nm}$. The colocalization analysis and determination of Pearson's coefficient were done using the WCIF ImageJ intensity correlation analysis plugin (http://wwwfacilities.uhnresearch.ca/wcif/ imagej) [25]. Images acquired were further processed using Adobe Photoshop, version 7.

\section{Variable-angle epifluorescence microscopy}

Four to 5-day-old Arabidopsis seedlings were mounted and observed under an inverted Olympus IX71 microscope equipped with the Andor TIRF illuminator. For sample preparation, seedlings were immersed in $1 / 2$ MS on a slide (BRAND Gmbh, Wertheim, Germany; $n$, $1.52 \pm 0.01$; thickness, $0.13-0.17 \mathrm{~mm})$. Another cover glass was placed on top of the sample, and this sandwich was pressed gently to tightly attach the seedling to the glass surface. The Andor attachment, which lies upstream of the objective, consists of a set of adjustable reflective mirrors controlling the path of the laser into the $100 \times$ oil-immersion TIRF objective (Olympus; numerical aperture $=1.45)$. The $473-/ 561-\mathrm{nm}$ laser line from a diode laser (Changchun New Industries Optoelectronics Technology) was employed to excite GFP and mCherry fluorophores respectively. Fluorescence 
signals were collected by the objective and passed through two filters, a BA 510IF long-pass filter (Chroma) and a HQ525/50 band-pass filter (Chroma), before being directed using a back-illuminated EMCCD camera (ANDOR iXon DV8897D-CS0-VP, Andor Technology) and highquality filters (band-pass 525/545 and 609/654 nm). We set the EM-gain of EMCCD camera at 433 throughout all imaging experiments unless otherwise indicated. Images were acquired with 100-ms exposure time (unless otherwise indicated) and analyzed with Image J software (NIH). The incident angle was adjusted and calibrated according to the methodology reported by Wan et al. [10], during the experiments, and the details were also included in Additional file 1. The co-localization analysis for dualcolor observations was performed by using WCIF ImageJ intensity correlation analysis plugin (http://www.facilities. uhnresearch.ca/wcif/imagej/colour_analysis.htm) [25].

Skewness analysis was used to obtain the extent of bundling, and the filament density was calculate as the percentage of occupancy of GFP-fABD2 signal in each micrograph [26, 27]. Micrographs were analyzed with ImageJ (http://rsb.info.nih.gov/ij/) using Higaki's macro available at $[26,27]$.

\section{Pharmacological studies with inhibitors}

TyrA23 and isobaxen were purchased from SigmaAldrich. The inhibitors were dissolved in 100\% DMSO as stock solutions and further diluted in 1/2 MS for VAEM imaging on epidermal cells. The final concentration of DMSO was $0.1 \%$ or even lower in all working solutions. Four- to 5 -d-old vertically grown seedlings were transferred from 1\% agar plates to a well of a 12-well plate containing $4 \mathrm{~mL}$ of final working concentration in $1 / 2$ MS. After the indicated incubation duration, seedlings were transferred to a glass slide with $100 \mu \mathrm{L}$ inhibitor solution, covered with a glass coverslip for imaging as above.

\section{Single particle tracking and data analysis}

Single-particle tracking was accomplished using spatially and temporally global particle assignment, with MATLAB as detailed in $[28,29]$, and only tracks with lengths of more than ten frames were kept for further analysis. For each trajectory, the mean square displacement (MSD) was computed from the formula:

$$
\operatorname{MSD}(\mathrm{t})=\frac{1}{\mathrm{~L}-\mathrm{n}} \sum_{\mathrm{s}=0}^{\mathrm{L}-\mathrm{n}-1}(\mathrm{r}(\mathrm{s}+\mathrm{n})-\mathrm{r}(\mathrm{s}))^{2}
$$

Where $n=t / \Delta t$, L is the length of the trajectory (number of frames) and $r(s)$ is the two-dimensional position of the particle in the frame $s$ ( $s=0$ corresponds to the start of the trajectory) [30]. To determine the diffusion coefficient from a trajectory, a line was fit to the MSD with $n$ running from 1 to the largest integer less than or equal to $L / 4$ [31]. The diffusion coefficient of the particles was calculated by linear fitting $(\mathrm{MSD}=4 \mathrm{Dt}+\mathrm{c})$ to $\mathrm{MSD}$ versus time (MSD-t), and the distribution of the diffusion coefficients was plotted in a histogram with logarithmically spaced bins. The acquired data were further subjected to Gaussian fitting, the position of the peak being considered the characteristic diffusion coefficient of each population. Quantification of the colocalization of CLC and AtFLOT1a was performed according to the protein proximity index method [29, 32, 33].

\section{Fluorescence correlation spectroscopy (FCS)}

FCS analysis was carried out on a Leica TCS SP5 FCS microscope equipped with a 488-nm argon laser, inhouse coupled correlator and Avalanche photodiode, using the point-scanning mode. The laser focus was placed at the plasma membrane of the cells, thus the fluctuations in fluorescence intensity was recorded during the diffusion of SKU-GFP molecules in and out the focal volume. Afterwards, the SKU5-GFP density was calculated according to the procedures described previously [4].

\section{Results}

VAEM is ideal for dissecting organellar identities and dynamics

Since the PM and intracellular compartments comprise a functionally interrelated network, we were keen to investigate the robustness for VAEM in tracking the dynamics of endomembrane components. In this regard, we studied auxin binding protein 1 (ABP1). Initially identified by its capacity to bind auxin and affect PM hyperpolarization, the functions of ABP1 are still under debate [34]. To obtain experimental cues for investigating the subcellular localization of ABP1, we generated an ABP1-YFP transgenic line under the control of its native promoter and examined the localization of ABP1 in the cotyledons under LSCM and VAEM, respectively. In accordance with previous reports, as visualized under LSCM, ABP1 was mainly localized to the PM and to highly dynamic intracellular structures (Fig. 1a). Unfortunately, it was not easy to discern under LSCM the intracellular structures that were labeled with fluorescence, but under VAEM it was reasonably clear that they were associated with the endoplasmic reticulum (ER) and some mobile intracellular compartments. As the expression level under the native promoter was low, the images obtained with wide-field epifluorescence could not clearly differentiate between areas with or without fluorescence (Fig. 1b). In contrast, we were gratified to discover that the intracellular compartments defined by ABP1-YFP fluorescence were well correlated with the mCherry-HDEL tagged ER in the time-series, they were 
(A)
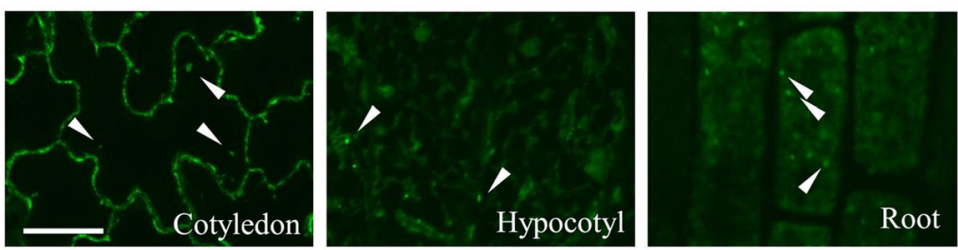

(B)
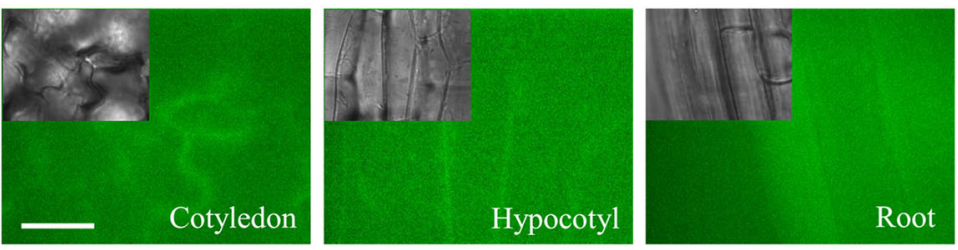

(C)
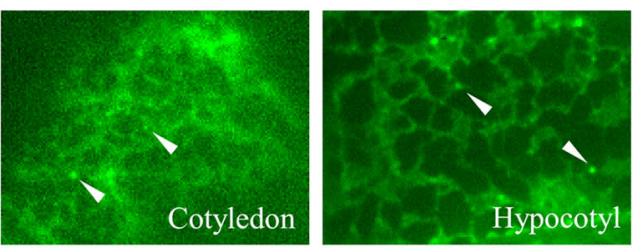

(D)
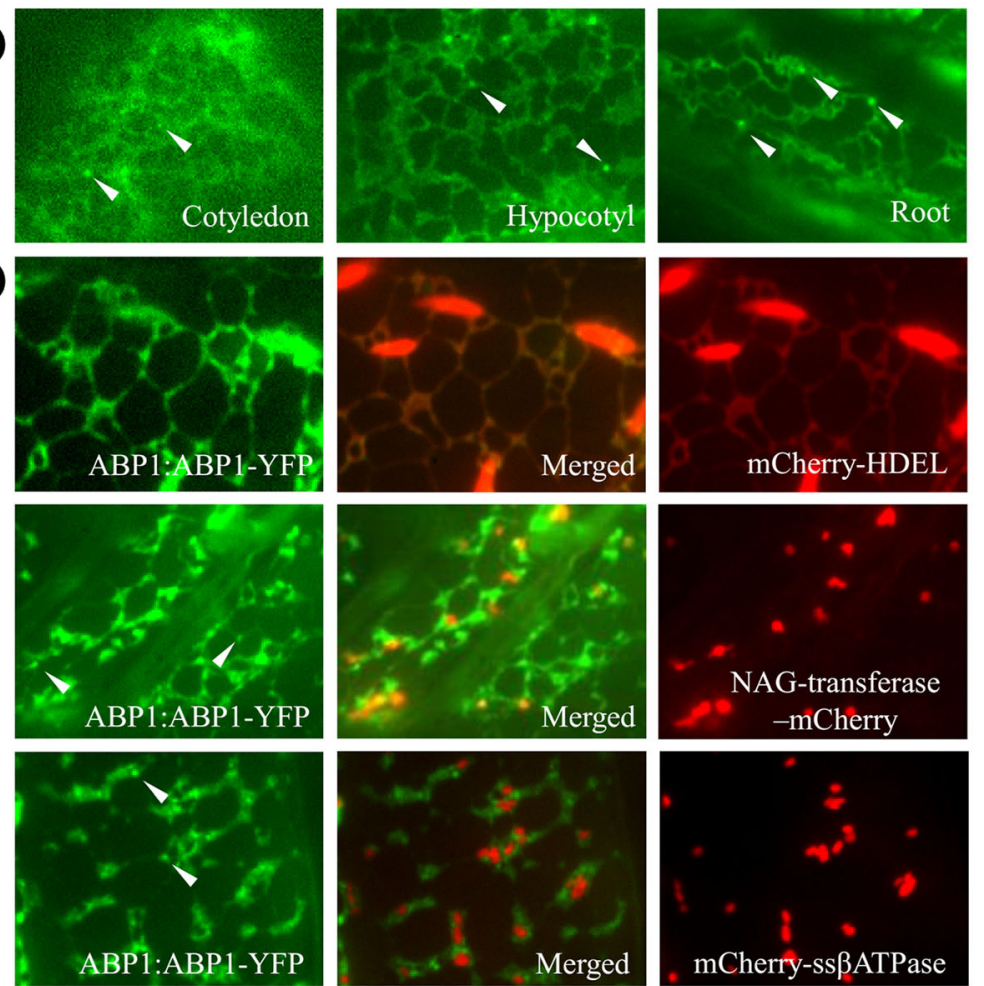

(E)
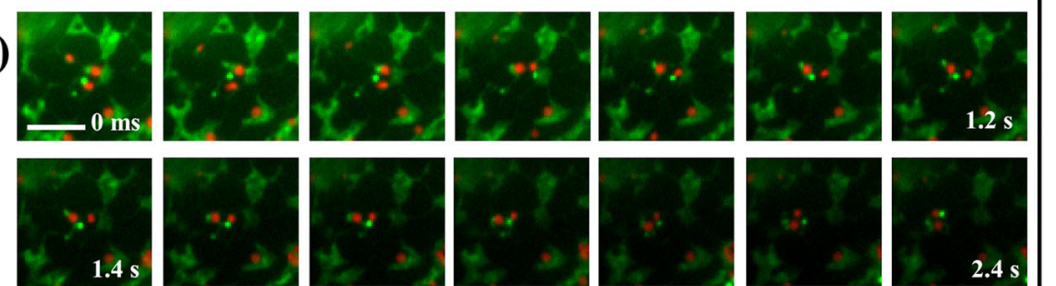

Fig. 1 Dual-color VAEM fluorescence co-localization with organelle markers is ideal for dissecting protein localization and dynamics in the cell cortex taking Auxin Binding Protein 1 (ABP1) as an example. The punctate structures in VAEM images were tracked by using spatially and temporally global particle assignment with MATLAB. The incident angles were between $63.03^{\circ}$ and $66.64^{\circ}$ for HDEL-GFP seedlings, corresponding to penetration depths at $120 \mathrm{~nm}\left(66.64^{\circ}\right)-250-300 \mathrm{~nm}\left(63.03^{\circ}\right)$ respectively, depending on the practical conditions for the adherence of seedling. a Transgenic Arabidopsis line expressing ABP1:ABP1-YFP (ABP1:ABP1-YFP / Col-0) showing subcellular localization to plasma membrane and intracellular structures, circles indicates punctate structures tagged by YFP fluorescence; $\mathbf{b}$ Transgenic Arabidopsis line expressing ABP1:ABP1-YFP showing blurred fluorescence, corresponding bright field images were shown in the inlets; c Transgenic Arabidopsis line expressing ABP1:ABP1-YFP showing uniformed distribution to ER and punctate structures under VAEM; $\mathbf{d}$ The punctate structures partly co-localize to Golgi apparatuses but do not co-localize to mitochondria; e Some ABP1-YFP tagged punctate structures frequently co-diffuse with Golgi apparatuses. The time series are shown for every 4 images. Bars $=200 \mu \mathrm{m}$ (a), $7 \mu \mathrm{m}$ (b-d), $5 \mu \mathrm{m}$ (e). Interval between frames $=200 \mathrm{~ms}$ 
partly co-localized with Golgi apparatuses (N-acetyl glucosaminyl (NAG) transferase-mCherry), but not with mitochondria markers (mCherry-ss $\beta A T P a s e)$ (Fig. 1c-d). Notably, some bright punctate structures, possibly vesicles or other endosomal structures, could be observed adjacent to the ER or transported along the extended ER tubules (Fig. 1d), further implying substantial roles for these ABP1-labelled structures in ER-endosome association. It was also found that the punctate structures codiffused with Golgi apparatuses in the time series (Fig. 1e), further suggesting the involvement of ABP1 in endosomal trafficking and endocytic/exocytic activities. As shown in Fig. 1, under VAEM the low background fluorescence allowed us to follow easily at high temporal resolution (200 ms) the ER dynamics in cotyledon epidermal cells as characterized using fluorescence proteins, which was not possible using conventional LSCM. Similarly, other functionally correlated organelles displaying high mobility, namely Golgi apparatuses and mitochondria, were also visualized with NAG transferase-GFP and mCherryss $\beta A T P a s e$ transgenic lines (Additional file 2: Figure S1, Additional files 3 and 4) [35, 36].

In addition to the imaging in cotyledon epidermal cells, VAEM is also applicable to epidermal cells of other tissues. We further used the transgenic HDEL-GFP line to follow ER dynamics in hypocotyl epidermal cells, root epidermal cells, young rosette leaves and stomata respectively, further demonstrating the wide applicability of VAEM in plant cell researches. Similarly, hypocotyl epidermal cells were readily imaged due to their relatively big size and planar shape (Additional file 2: Figure S2). It can be found the ER tubules were distributed in a lower density in root. However, as part of the tubules were not in the imaging plane, the variable and non-planar shape of leaf pavement cells only allow us to observe ER dynamics in limited areas closely adhering to the slide in these cells (Additional file 2: Figure S1 and Additional file 5). As cell wall and specimens of larger sizes may inevitably pose a physical barrier in observations on plant cells, freshly detached tissues are always preferred to obtain better efficacy in adhering the specimen closely to the slide, which is particularly applicable to observations in cotyledon and young leaves.

\section{Endocytic events and intracellular trafficking can be followed under VAEM}

Given that, as the smallest units, endocytic vesicles play pivotal roles in connecting different endosomal components, the role of clathrin in internalizing extracellular substances has been well investigated, as it is assembled at the PM and initiates endocytic events [37]. In the present study, the TIRF system was equipped with a micrometer allowing continuous lateral adjustment of the spatial filter assembly, such that the position of the beam at the back aperture of the objective could be modulated to switch between TIRF and epifluorescence modes. Under epifluorescence illumination, only blurred fluorescence was found to be associated with the PM, whereas, under VAEM, clathrin light chain (CLC)-GFP formed discrete foci at the PM or were organized into small clusters, contributing to the formation of an endocytic complex at the site (Fig. 2a and Additional file 2: Figure S3). The CLC-GFP foci at the PM could be easily distinguished, though CLC-GFP may also label some brighter, out-of-focus intracellular structures, corresponding to different incident angles and penetration depths [ranging from approximately $100 \mathrm{~nm}\left(67.73^{\circ}\right)$ and $\left.130 \mathrm{~nm}\left(65.69^{\circ}\right)\right]$ (Additional file 2: Figure S3 and Additional file 1, indicated with arrowhead), possibly Golgi apparatuses or trans-Golgi network (TGN). When the incident angle of the laser was decreased from subcritical angles towards zero degrees, the foci were lost in the background signal arising from organelle-localized CLC-GFP and cytoplasmic CLC-GFP signals. Similarly, AtFLOTTILIN1a (FLOT1a) is identified as another candidate involved in endocytic activities independent of the clathrin-mediated pathway [14]. As it has been reported previously to be a potential raft-located protein [14], the dynamics of FLOT1a were also examined under VAEM. As expected, mCherry-FLOT1a was found to be prominently associated with the PM and was organized into punctate structures. The FLOT1a-positive punctate structures were dynamic in the vicinity of the PM, but in contrast to those observed in the CLC-GFP lines, some of the puncta were laterally mobile (Fig. 2c and Additional file 6), possibly as individual structures budding off from the PM and entering the cytoplasm. In the presence of BFA, a specific pharmacological agent capable of inhibiting COPI vesicle formation at the Golgi apparatus [38], CLC-GFP and mCherry-FLOT1a were largely colocalized to the BFA-induced compartments during their formation, whereas there were still some apparently associated green and red puncta in the cytosol (Fig. 2d).

As both CLC-GFP and mCherry-FLOT1a are sensitive to brefeldin A (BFA) treatments (Fig. 2a-b), we were curious to examine whether CLC-coated vesicles and FLOT1a-tagged vesicles were colocalized following internalization. Therefore, fluorescence-based co-localization analysis was employed to study the functional correlation of both proteins in terms of their specific cellular location. We co-expressed CLC-GFP and mCherry-FLOT1a and then analyzed their spatial correlation using LSCM images and VAEM time-series images, respectively. Both FLOT1a and CLC have been reported previously as membrane associated proteins and, as shown in Fig. 2a, both CLC-GFP and mCherry-FLOT1a were found to be localized to the PM and to some intracellular structures. Further close-up LSCM observations indicated that CLC-GFP 


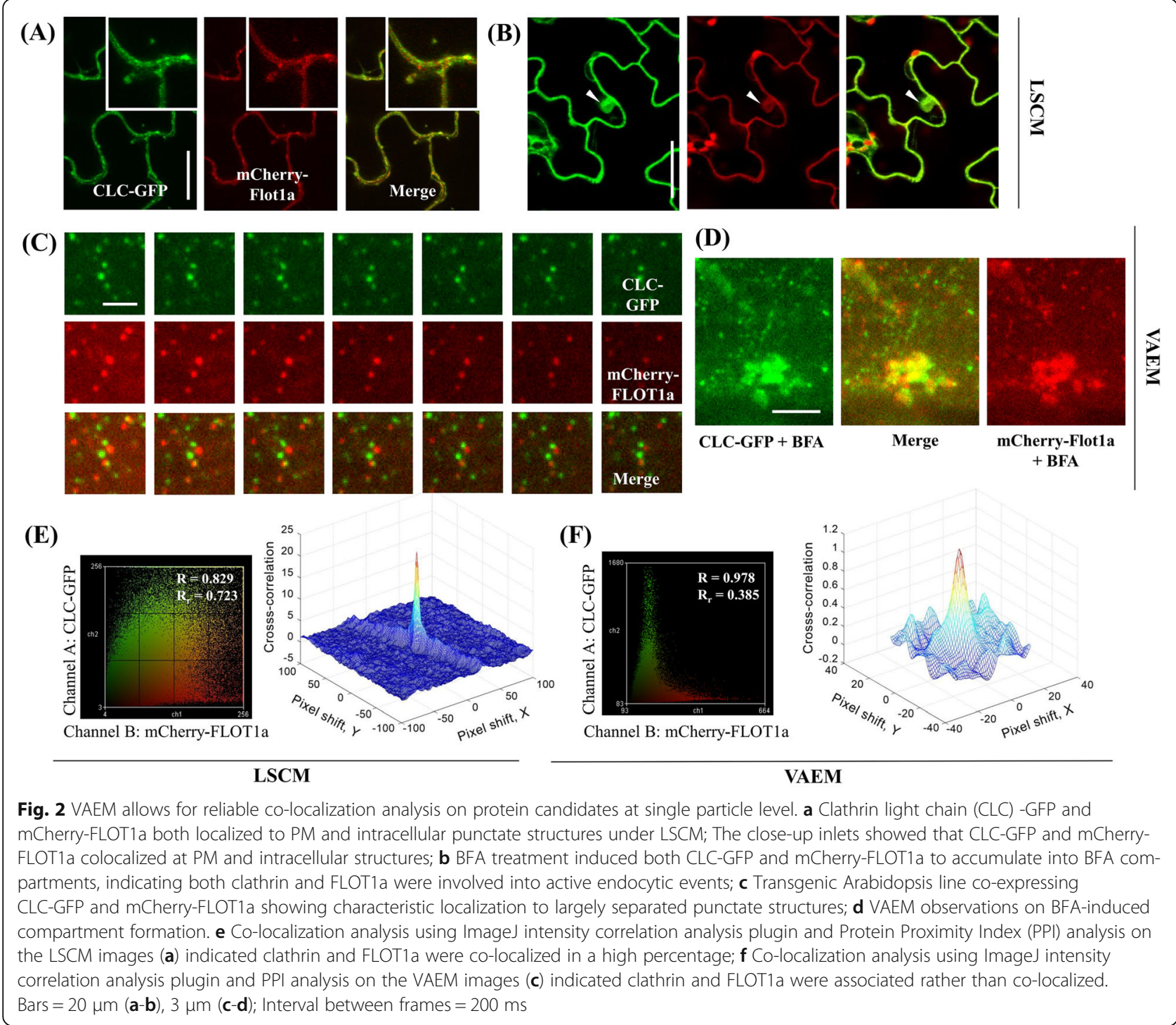

and mCherry-FLOT1a puncta were co-localized or closely associated with each other, correlating well with the high overlap coefficient, $R_{r}=0.723$, as shown in the scatter plots (Fig. 2a and e). In contrast to the LSCM findings, however, the VAEM time series showed that in fact most of the CLC-GFP and mCherry-FLOT1a puncta were not co-localized (Fig. 2c). As a result, the overlap coefficient $\mathrm{R}_{\mathrm{r}}$ was 0.385 from the calculation using the time-series images (Fig. 2f, ten sequential frames in the present study), indicating a low percentage of co-localization of these two protein candidates and further suggesting that VAEM time-series can provide a more reliable co-localization analysis of intracellular structures. As a quantitative measure of colocalization emphasizing that colocalization occurs at the length scale of the resolution of the microscope [33], Protein Proximity Index (PPI) was also calculated for comparison, as shown in the $3 \mathrm{D}$ cross-correlation plots (Fig. 2e and f), [PPI $=0.25 \pm 0.05$ (GFP channel) and $0.41 \pm 0.06$ (mCherry channel) for VAEM, where $\mathrm{PPI}=0.53 \pm 0.17$ (GFP channel) and $0.72 \pm 0.22$ (mCherry channel) for LSCM]. Moreover, transgenic Arabidopsis lines co-expressing CLC-GFP / mCherry-FLOT1a, GFPFLOT1a / mCherry-FLOT1a and CLC-GFP / dynamin related protein $1 \mathrm{C}$ (DRP1C)-mOrange generated by crossing, were each subjected to VAEM observations followed by single-particle tracking (SPT) and calculations were made of the distances between captured dual-color labeled punctate structures (Fig. 3a). In the control (GFP-FLOT1a and mCherry-FLOT1a), about $50 \%$ of the GFP signal was classified as co-localized and more than $40 \%$ was classified as associated. Among the protein candidates examined, CLC were found to be either co-localized (about $30 \%$ ) or associated (50\%) closely with DRP1C, 
(A)

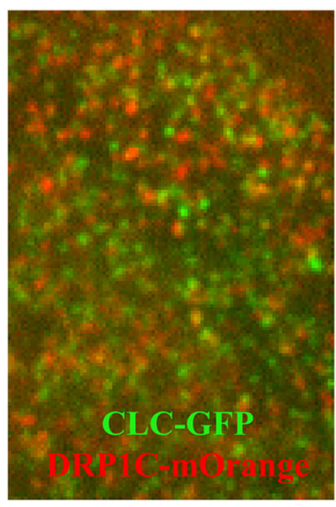

(B)

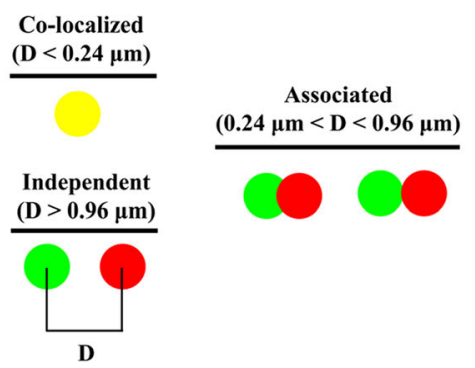

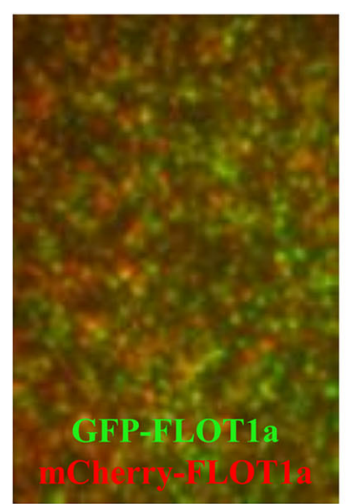

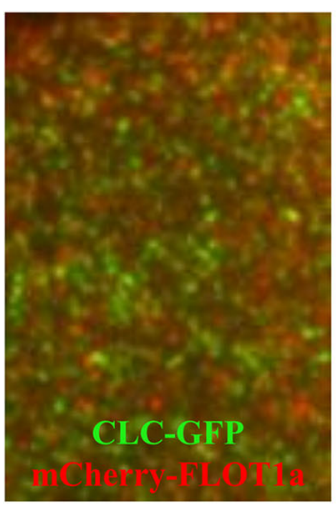

(C)

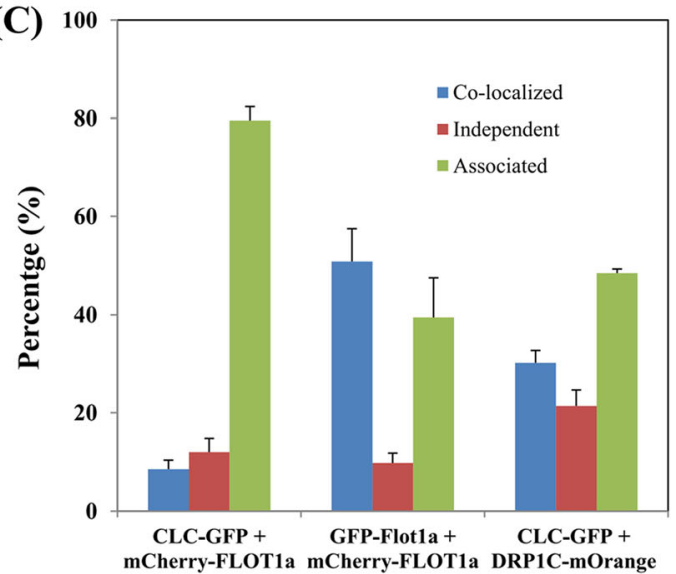

Fig. 3 Co-localization analysis on proteins of interest using VAEM-based single particle tracking. a Transgenic Arabidopsis line CO-expressing CLCGFP / DRP1C-mOrange, GFP-FLOT1a / mCherry-FLOT1a and CLC-GFP / mCherry-FLOT1a were subjected to VAEM followed by single particle tracking and calculation on the distance between the captured punctate structures labelled by dual colors. $\mathbf{b}$ We classified the resulting distances into three categories: (i) colocalized: a distance between two centers that was below the resolution limit of the objective lens $(0.24 \mu \mathrm{m}$ in this study); (ii) associated: a distance less than the sum of the radii of two punctate structures ( $<0.96 \mu \mathrm{m}$ in this study); and (iii) independent: a distance larger than the sum of the radii of two punctate structures $(>0.96 \mu \mathrm{m})$. D stood for the distance between the centers of two adjacent puncta for analysis. c Percentages of co-localized, associated and independent puncta for the protein candidates

which was consistent with their synergistic actions in vesicle formation and internalization; furthermore, about $80 \%$ of the CLC-positive puncta were classified as associated with mCherry-FLOT1a, whereas only $8 \%$ or $12 \%$ of the GFP signals, respectively, was co-localized to or independent to mCherry-FLOT1a puncta, further confirming the applicability of VAEM-based SPT for the co-localization analysis of putatively correlated proteins (Fig. 3b-c).

\section{VAEM observations on coordinate behaviors in cytoskeletal dynamics}

Luckily, VAEM imaging using subcritical angles also allowed the tracking of cortical actin filaments (AFs) labelled with the GFP-tagged F-Actin Binding Domain of Fimbrin2 (GFP-fABD2) (Fig. 4, Additional file 2: Figure S4, and Additional file 7). As shown in Additional file 2: Figure S4, extensive arrays of longitudinal actin bundles and a dynamic network of AFs could be observed in elongated epidermal cells of the hypocotyls. Several filaments rapidly became bundled into single filaments, but also individual filaments participated in the creation of multiple bundles, all utilizing the same zipper-like motion (indicated by arrows). To follow the dynamics of microtubules (MTs) in epidermal cells, epidermal hypocotyl cells of plants expressing mCherry- $\alpha$-tubulin 5 isoform (TUA5) were also imaged. The MTs were clearly visible at subcritical angles, showing all the typical configurations previously described for wild-type seedlings: basket, longitudinal, oblique and transverse array (Additional file 2: Figure S5). In time-lapse images of mCherryTUA5-expressing cells (Additional file 8), though microtubule growth was apparent, microtubules did not show obvious assembly and disassembly events at a temporal resolution of $200 \mathrm{~ms}$.

Simultaneous tracking of both types of cytoskeletal components using stably transformed lines with dualcolor reporters may provide a powerful tool for revealing 


\section{(A)}
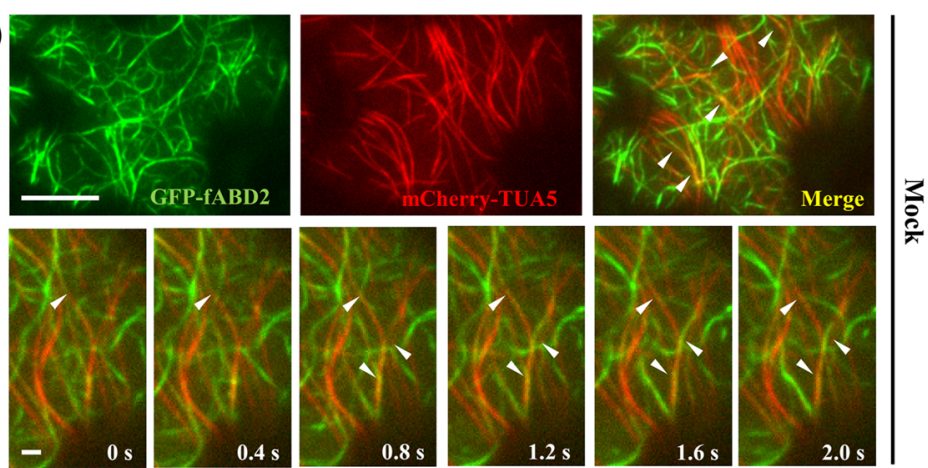

(C)
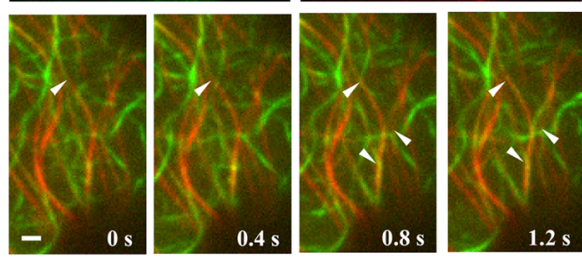

$\frac{1}{3}$

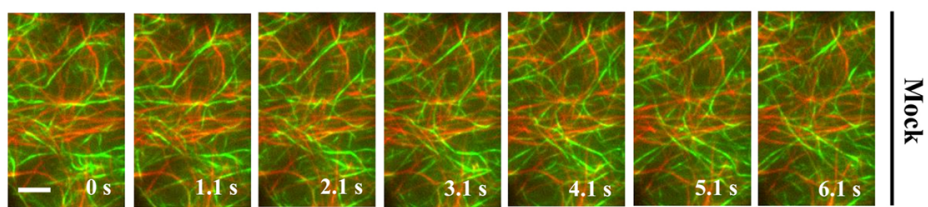

(D)
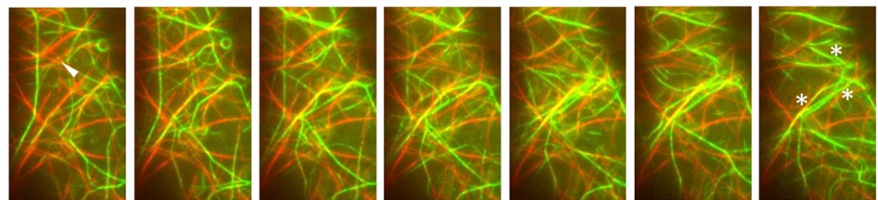

运

(E)
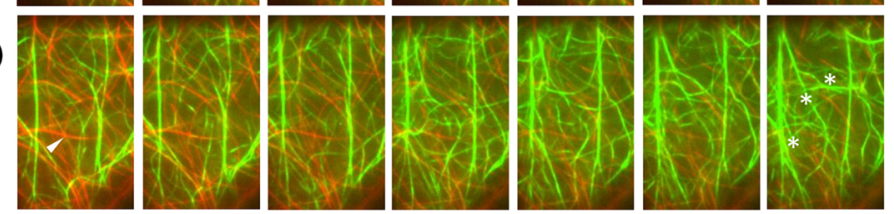

(F)
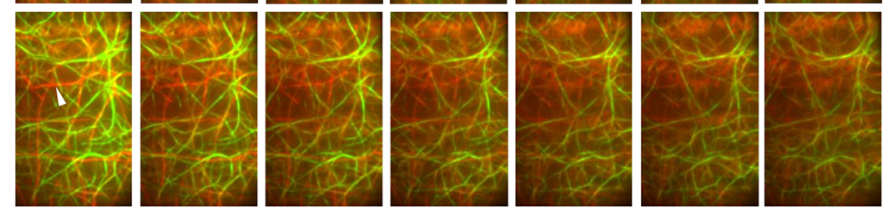

$\frac{2}{3}$
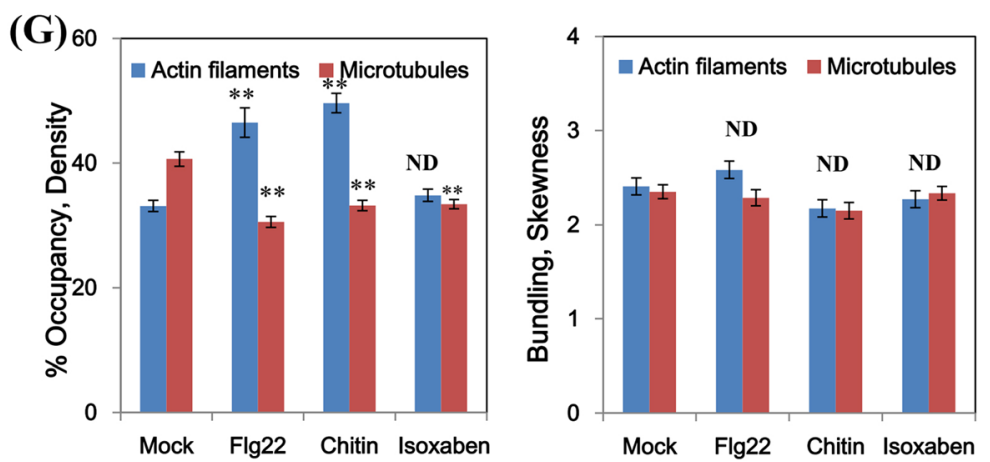

Fig. 4 (See legend on next page.) 


\begin{abstract}
(See figure on previous page.)
Fig. 4 PAMP significantly altered cytoskeletal architecture and dynamics as revealed by dual-color VAEM. To investigate whether the two cytoskeletal structures interact coordinately, seedlings expressing green fluorescent protein (GFP)-F-actin binding domain of fimbrin2 (fABD2) and mCherry-atubulin 5 isoform (TUA5) were generated by crossing. LSCM and VAEM were carried out on the seedlings respectively. a AFs and MTs in mock-treated epidermal cells from the wild-type cells, arrows indicate actin fragments reside in transient coincidence with MTs; $\mathbf{b}$ Selected frames from a time series of a GFP-fABD2 and mCherry-TUA5 dual-labeled line; c AFs and MTs in the wild-type epidermal cells. $\mathbf{d}$ to e AFs appeared to be more abundant after flg22 and chitin treatment, while the transverse MT organization gradually changed to longitudinal fragments. Epidermal cells treated with $1 \mu \mathrm{M}$ flg22 or $10 \mu \mathrm{M}$ chitin had a significant increase in AF abundance compared with mock-treated seedlings. $\mathbf{f}$ To address the specificity for the elicitortriggered reorganization of cytoskeletal components as above, $50 \mu \mathrm{M}$ isoxaben, a cellulose synthesis inhibitor, was applied to treat the seedlings. The MTs were dramatically disordered following incubation while AFs did not show significant changes in density and intensity. $\mathbf{g}$ Average filament density (percentage of occupancy) analysis was performed on images collected from epidermal cells in the cotyledons. When compared with mock-treated cells, the AF density was significantly increased after $1 \mu \mathrm{M}$ flg22 and $10 \mu \mathrm{M}$ chitin treatments; in contrast, MT density decreased significantly after chitin and flg22 treatment, while isoxaben treatment significantly decrease MT density without affecting AF density; The extent of filament bundling, or skewness, was measured. No significant differences were observed among treatments. Values given were mean \pm SE. $(n=100$ cells from 10 cotyledons per treatment; ** $P<0.001$; ND, no significant difference; Student's t-test). Bars $=10 \mu \mathrm{m}$. Interval between frames $=200 \mathrm{~ms}$, "0 $\mathrm{s}^{\prime \prime}$ referred to the time point at 5 min after corresponding treatment
\end{abstract}

coordinate behaviors and elucidating underlying mechanisms. VAEM observations on the dual-labeled epidermal cells demonstrated that cortical AFs and MTs were coaligned at numerous sites (Fig. 4a-b), which were mainly found between transversely or obliquely oriented AFs and MTs at the cell cortex. Straightening and bending events of cortical AFs, as reported previously [39], were also occasionally observed to occur within a couple of seconds at coalignment sites (Fig. 4b). Several studies have shown that perturbations of one cytoskeletal component by extracellular stimuli can change the organization of the other [18]. Here, we observed that the transverse MT organization gradually changed to a disordered configuration upon Pathogen-Associated Molecular Pattern (PAMP) (flg22 and chitin) exposure and resulted in a reduction of co-alignment between AFs and MTs (Fig. 4d-e). To quantify cytoskeletal remodeling in cotyledons, the cortical actin architecture was measured for density and skewness, which are metrics used for cytoskeletal component to estimate the percentage of occupancy and the extent of bundling, respectively $[26,40]$. As shown in Fig. 4d-e and g, AF density in the cortical array was significantly increased after 5 -min treatment with $1 \mu \mathrm{M}$ flg22 or $10 \mu \mathrm{M}$ chitin $(P<0.001)$, which was consistent with previous results for dark-grown hypocotyl cells [40]. However, MTs showed a significant decrease in density in the focus plane $(P<0.001)$, further suggesting that the MTs may also play a specific role in the perception of pathogenic microbes. Collectively, these data demonstrate that the cortical actin array in cotyledon epidermal cells responds within minutes to several diverse PAMPs, leading to significant increases in AF density. To examine the response specificity for the elicitor-triggered reorganization of cytoskeletal components as above, isoxaben, a cellulose synthesis inhibitor, was applied to the seedlings. As expected, following incubation the MTs were dramatically disordered and were changed into almost longitudinal fragments, further confirming the microtubule / microfibril paradigm; in contrast, actin filaments did not show significant changes in intensity and density $(P>0.05)$ (Fig. 4f-g). These results address the specificity of the coordinated behavior of AFs and MTs in response to PAMP elicitation, though the underlying mechanisms need further investigations.

\section{Comparative analysis of membrane compartmentalization by using LSCM and VAEM}

As with yeast and animal cells, plant cells similarly have a sub-compartmentalized PM, in which membrane microdomains have been demonstrated to modulate endocytic events and signaling initiation [24, 41]. As shown in Fig. 5, transgenic Arabidopsis seedlings expressing several previously identified microdomain-located proteins (StREM1.3, AtREM1.2 and AtFLOT1a) were generated and examined under LSCM and VAEM, respectively. Two well-characterized glycosyl phosphatidylinositol (GPI)-anchored proteins, SKU5 and COBRA, were also selected, as it has been extensively reported in animal and in yeast cells that GPI proteins are predominantly located in membrane rafts $[20,42]$. To obtain a detailed view of the membrane domains, LSCM was used to image the upper surface plane of cotyledon epidermal cells of 3- to 4-day-old plants. A diffuse but uniformly distributed fluorescence labeling of the PM was observed for the two previously identified remorins (REMs) and for the GPIanchored proteins, however, microdomains showing significantly increased fluorescence intensity were not detected (Fig. 5b-f), while AtFLOT1a-GFP displayed an uneven localization on the cell surface (Fig. 5a).

By contrast, as VAEM allows observations at high signal-to-noise ratio (SNR) for cellular events occurring in the vicinity of the PM, both AtREM1.2 and StREM1.3 segregated into distinct microdomains showing relatively low motility in the time-lapse images as expected (Fig. 5b-d). Notably, GFP-AtREM1.2 as expressed under the control of the endogenous promoters formed static punctate structures as seen under VAEM (Fig. 5c-d), 


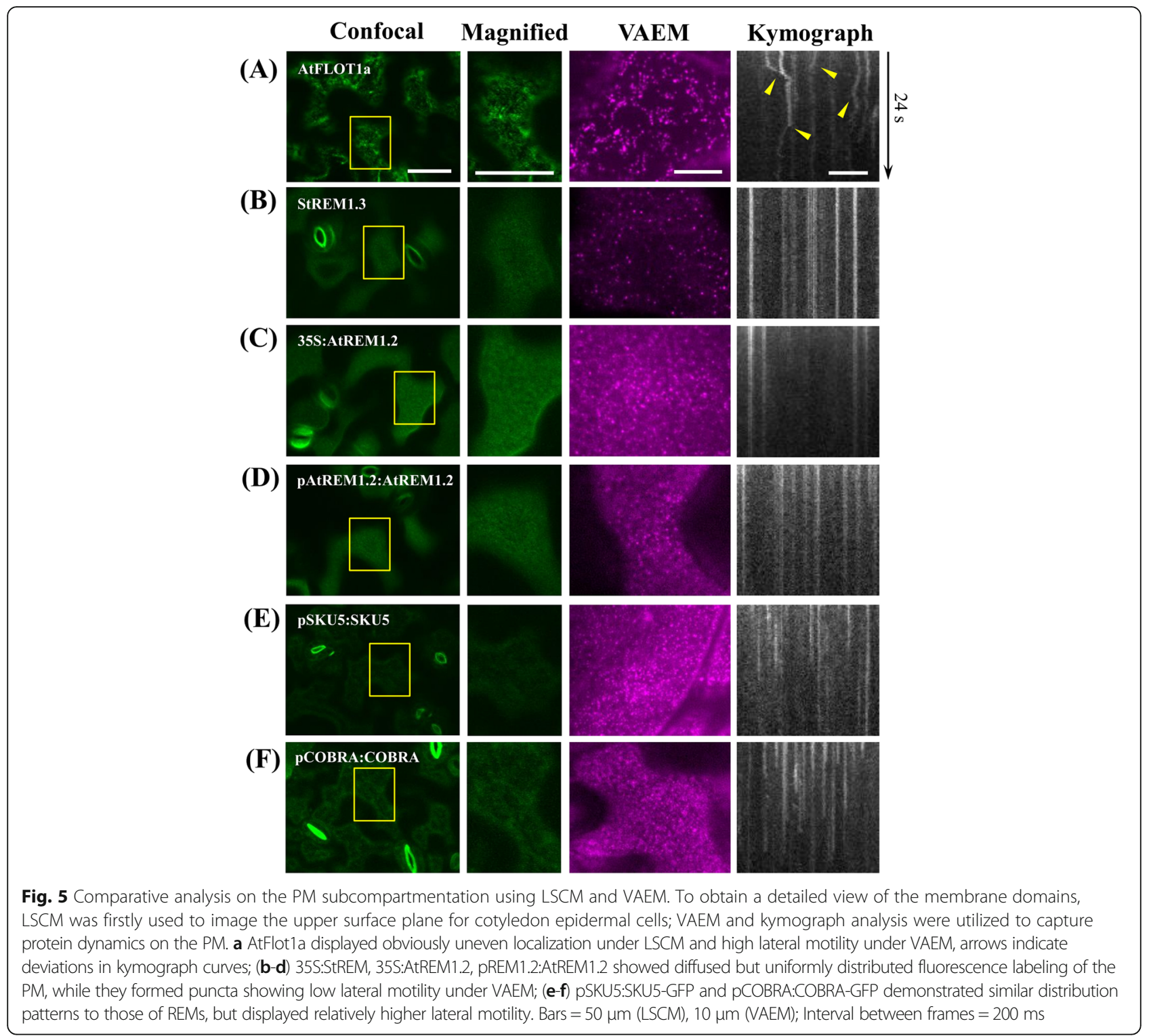

which were not significantly different from that expressed under the control of $35 \mathrm{~S}$ promoter, indicating that AtREM1.2 overexpression did not significantly change the expression pattern or the signal intensity in the membrane microdomains. To assess the temporal stability of these microdomains, time-lapse recording and kymograph analysis were performed on the timeseries images. As expected, both SKU5 and COBRA displayed similar patterns in fluorescence distribution and punctate motility in comparison to REMs, since the results clearly showed that, for most of the punctate structures, REMs, SKU5, and COBRA all exhibited undetectable lateral stability (Fig. 5e-f). Interestingly, another previously identified microdomain-located protein, AtFLOT1a-GFP, displayed high motility and showed variability in punctate sizes, which may correspond to endocytic vesicles or different oligomerization statuses as previously reported for their homologs in mammalian cells (Fig. 5a) [14]. It did not localize to relatively stable microdomains in the PM, unlike StREM1.3 and AtREM1.2 as described above, further implying the heterogeneity of distinct microdomains and the biological complexity of membrane compartmentalization in vivo.

Kinetic properties of membrane proteins as resolved by VAEM and single-particle tracking

The PM provides sufficient spaces within which macromolecular interactions can take place efficiently, including the clustering of proteins in oligomeric complexes and the lateral diffusion of proteins. So far, among the currently identified GPI-anchored proteins in plant cells, SKU5-GFP has been experimentally demonstrated to be associated 
with the PM and localized to intracellular structures [20], which were found to be well co-localized with FM4-64stained PM and vesicles (Additional file 2: Figure S6). The motility of membrane proteins is a critical determinant of their interaction capabilities and functions. Utilizing VAEM-based single-particle tracking, we were able to record and analyze the kinetic parameters representing the dynamics of SKU5-GFP puncta at single-particle level, including the fluorescence intensity, retention time, motion range, velocity, trajectory, and diffusion coefficient of individual puncta. As an example, the fluorescence intensity and retention time of individual puncta were analyzed with MATLAB software as examples (Fig. 6).
Notably, the SKU5-GFP puncta displayed different types of dynamic behavior, in which the fluorescence intensity and the retention time were two major parameters for characterizing the motility and oligomeric status of this GPI-anchored protein (Fig. 6a-b). The cumulative histograms for fluorescence intensity showed a skewed asymmetric distribution $(n=1813)$. Two-fifths $(40.77 \%)$ of the particles had intensities ranging from 100 to 500 a.u., approximately the range for diffraction-limited monomeric GFP molecules in control cells (Additional file 2: Figure S7), suggesting that these particles were mostly composed of SKU5-GFP monomer. In contrast, the remaining puncta can be considered to exist as

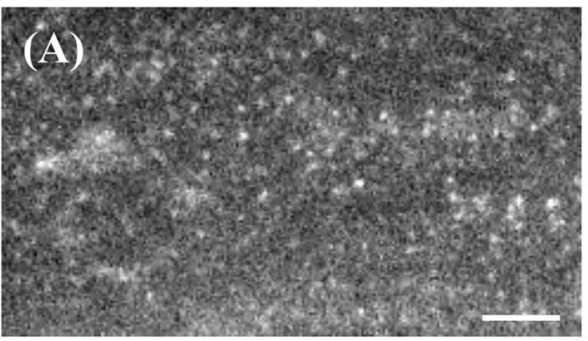

(C)

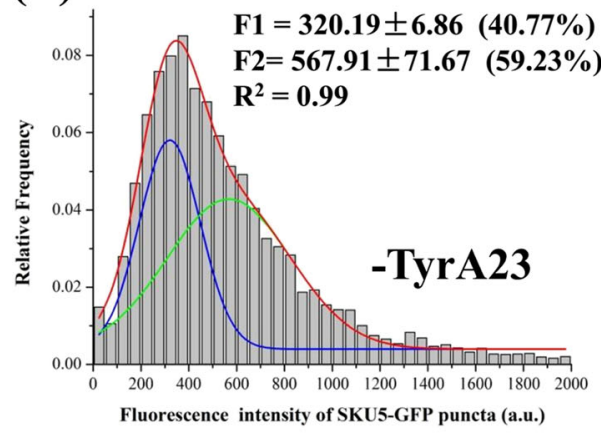

(E)

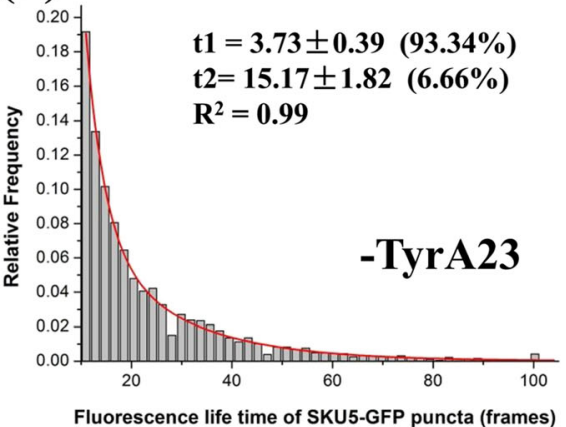

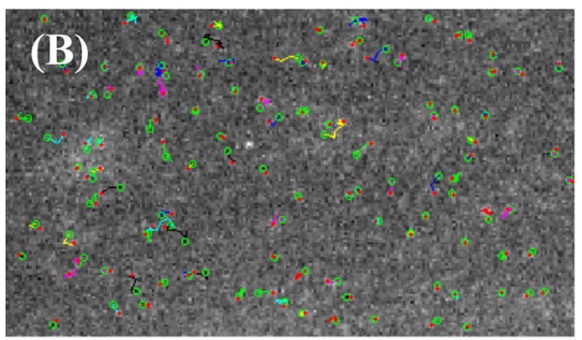

(D)

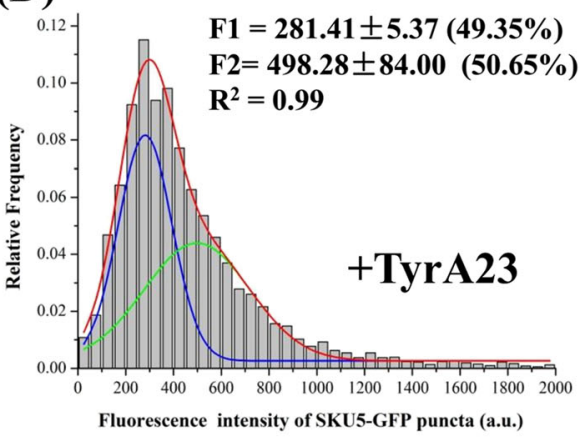

(F)

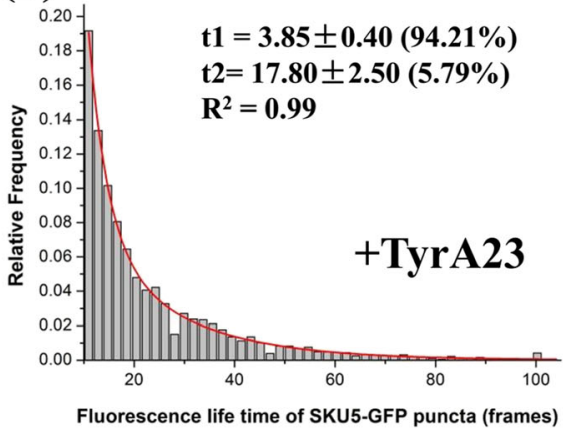

Fig. 6 Single particle tracking and computational analysis on puncta of SKU5-GFP captured with VAEM. a A representative frame of time-lapse images for SKU5-GFP tagged puncta; $\mathbf{b}$ Trajectory of tracked puncta of SKU5-GFP-tagged puncta in panel (a); $\mathbf{c}$ The tracked SKU5-GFP tagged puncta were skewed to two populations in the histogram analysis based on fluorescence intensity after Gaussian fitting, implying different clustering pattern for the two populations; $\mathbf{d}$ TyrA23 treatment significantly altered the proportions of the two populations $(P<0.05)$; e The SKU5-GFP puncta were aligned to two populations in the histogram analysis based on life time after exponential fitting, implying different association states of the two populations; $\mathbf{f}$ The lifetime for two populations of SKU5-GFP puncta did not change significantly upon TyrA23 treatment $(P>0.05)$. Bars $=50 \mu \mathrm{m}$; $P<0.05$, student t-test was carried out to examine the significance for the differences in the proportions between the two populations of SKU5-GFP puncta after tyrA23 treatment; Interval between frames $=200 \mathrm{~ms}$ 
clusters composed of two or more SKU5 molecules since the cumulative histograms can be fitted into another Gaussian peak (59.23\%) (Fig. 6c). In addition, the retention time (represented by the number of frames) for these particles, from their appearance to their disappearance on the membrane surface, ranged from 10 to 70 frames ( $2.3 \mathrm{~s}$ to $16.5 \mathrm{~s}$ ), and the cumulative histograms of the lifetimes could be well fitted by a two-order exponential curve $(\tau 1=3.73 \pm 0.39,93.34 \% ; \tau 2=15.17 \pm 1.82$, 6.66\%) (Fig. 6e). Moreover, the fit was not further improved with an increase to three exponential components, further implying that, in terms of their lifetime on the PM, SKU5-GFP molecules were largely present in the form of monomers or were organized into oligomers. To test whether clathrin-mediated endocytosis plays a role in membrane dynamics of SKU5-GFP, given that SKU5-GFP colocalized with FM4-64 in intracellular structures, pSKU5:SKU5-GFP seedlings were treated with $50 \mu \mathrm{M}$ tyrphostin A23 (tyrA23), an inhibitor of clathrin-dependent endocytosis. It was found that, as a consequence, the proportions of the two subpopulations of pSKU5:SKU5-GFP puncta varied significantly $(281.41 \pm$ 5.37, 49.35\%; 498.28 $\pm 84.00,50.65 \%)(n=1760, P<0.05)$ compared to the control, while the retention time of SKU5-GFP puncta did not show significant changes ( $\mathrm{\tau} 1=$ $3.85 \pm 0.40,94.21 \% ; \tau 2=17.80 \pm 2.50,5.79 \%) \quad(n=1760$, $P>0.05$ ) (Fig. 6d and f), further implying that clathrinmediated internalization was involved in the membrane dynamics of both SKU5-GFP subpopulations. Importantly, TyrA23 significantly decreased the fluorescence intensity of both subpopulations and also the percentage of the subpopulation exhibiting higher fluorescence intensity (Fig. 6c-f). Further fluorescence correlation spectroscopy (FCS) analysis on fluorescence fluctuation also revealed a lower SKU5-GFP density $\left(38.4 \pm 4.1\right.$ molecules $\mathrm{mm}^{-2} ; 14.9 \%$ decrease with respect to control cells at $44.6 \pm 3.4$ molecules $\mathrm{mm}^{-2}$; $P<0.05$, t-test) was detected after treatment with TyrA23 (Additional file 2: Figure S8).

More than 25\% of the proteome of higher plants is predicted to be membrane-associated proteins, including integral membrane proteins or peripheral membrane proteins according to the difference in their topology [43] (Fig. 7a). Considering this, we further selected SKU5 and COBRA, BOR1 and LTi6a, FLOT1a and StREM1.3, which are, respectively, well-characterized representatives of GPI-anchored proteins in the outer leaflet of the PM, transporters as representatives of integral membrane proteins, and proteins associated with the inner leaflet of the PM through post-translational modifications (such as S-acylation) respectively. As all of these proteins exhibited similar punctate structures varying in fluorescence intensity under VAEM, we then studied their dynamic properties in more detail by SPT and further analyzed the dynamic parameters using MATLAB software.

The diffusion coefficient (DC) is obtained from the trajectory of an individual particle, and the statistical distribution of single-trajectory diffusion coefficients may be useful as a measure of the heterogeneity of the membrane (Additional file 2: Figure S9) [28]. Both SKU5 and COBRA are well-characterized GPI-anchored proteins in plant cells, and they are expected to be preferentially located in membrane microdomains [41]. As a result, the diffusion coefficients of SKU5 and COBRA were distributed within one subpopulation of diffusion behavior $\left(\mathrm{DC}=0.80 \times 10^{-3} \mathrm{\mu m}^{2} / \mathrm{s}, \quad n=1321\right.$, SE $0.77 \times 10^{-3}-$ $\left.0.83 \times 10^{-3} \mu \mathrm{m}^{2} / \mathrm{s}, \mathrm{r}^{2}=0.99\right)\left(\mathrm{DC}=1.35 \times 10^{-3} \mu \mathrm{m}^{2} / \mathrm{s}\right.$,

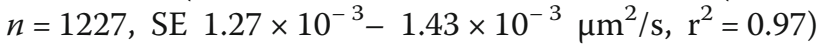
(Fig. 7b). Similar to SKU5, the DC for LTi6a-GFP puncta was skewed to $1.97 \times 10^{-3} \mu^{2} / \mathrm{s}(n=1228$, SE $1.86 \times 10^{-3}-2.08 \times 10^{-3} \mu \mathrm{m}^{2} / \mathrm{s}, \mathrm{r}^{2}=0.97$ ), which was comparable to the results obtained for the two GPIanchored proteins; in contrast, the bona-fide endocytic cargo transmembrane protein BOR1-GFP was also distributed in a punctate manner as observed by VAEM [19]. The DC was $2.60 \times 10^{-2} \mu^{2} / \mathrm{s} \quad(n=1013$, SE $2.49 \times 10^{-2}-2.71 \times 10^{-2} \mu \mathrm{m}^{2} / \mathrm{s}, \mathrm{r}^{2}=0.99$ ) (Fig. 7b), with a large dispersion of diffusion coefficients, most of which

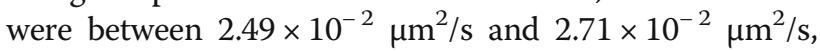
indicating that the motility of these proteins was heterogeneous, as also shown by kymograph analysis (Additional file 2: Figure S10). The resulting histograms were fitted by one or two Gaussian peaks, identifying as different subpopulations characterized by varying diffusion coefficients. In contrast, the inner-leaflet located AtFLOT1a displayed apparent high motility, and its DC was $1.21 \times 10^{-2} \mu \mathrm{m}^{2} / \mathrm{s}$ $\left(n=1137\right.$, SE $\left.1.14 \times 10^{-2}-1.29 \times 10^{-2} \mu^{2} / \mathrm{s}, \mathrm{r}^{2}=0.98\right)$, further implying its potential involvement in endocytic events as previously reported. It was notable that the DC for StREM1.3-GFP was the lowest for all of the protein candidates examined, which together spanned a relatively broad range from $10^{-8} \mu \mathrm{m}^{2} / \mathrm{s}$ to $10^{-2} \mu \mathrm{m}^{2} / \mathrm{s}$, with $1.13 \times$

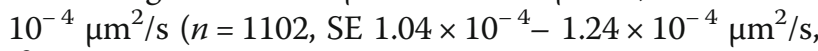
$\left.r^{2}=0.94\right)$ as the median value; this further implied low motility for StREM1.3-GFP puncta and substantial scaffolding functions in the maintenance of membrane microdomains and in the initiation of signaling processes, as previously reported.

\section{Discussion}

With recent advances in novel fluorescent proteins and imaging techniques, it is now feasible to visualize biological processes close to the PM at the subcellular level, or even at the single-particle level. TIRFM has been originally applied in plant research to in vitro studies on the AF and MT dynamics, as well as ER dynamics in protoplasts lacking cell wall [44]. Due to the exponential 

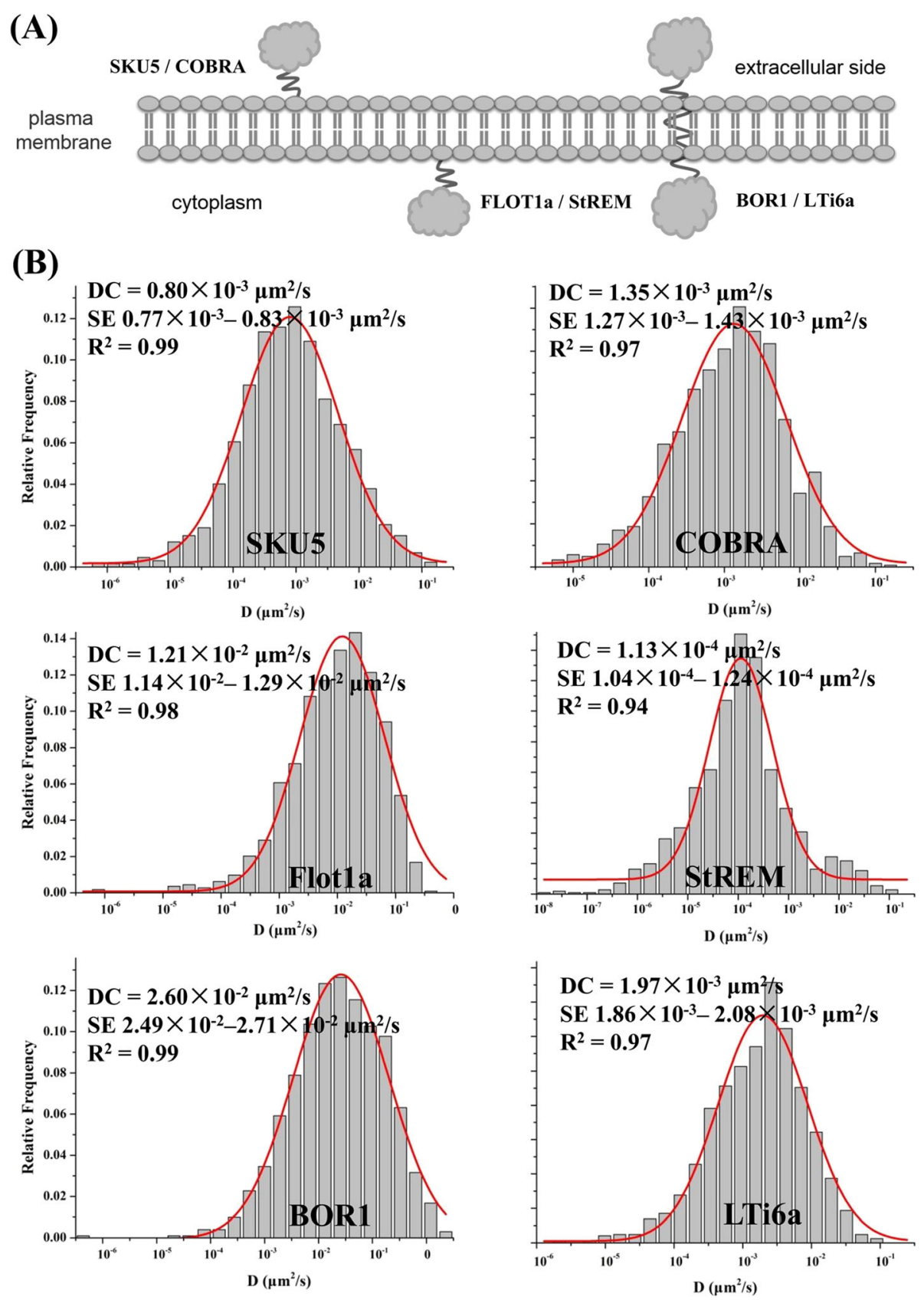

Fig. 7 VAEM allows for analysis on dynamics of individual protein candidate at single particle level. Transgenic Arabidopsis line expressing pSKU5:SKU5-GFP, pCOBRA:COBRA-GFP, pBOR1:BOR1-GFP, 35S:LTi6a-GFP, 35S:GFP-Flot1a and 35S:GFP-StREM showing characteristic localization to punctate structures (a), which allows for further analysis on their dynamics on the PM, characterized by diffusion coefficient (DC) (b)

decay of the evanescent wave produced, the application of TIRFM to the study of plant and fungal cells is not straightforward, which is hampered by thick cell walls and poor adherence to glass surfaces. VAEM is applicable to different types of epidermal cells in plants. The first application of VAEM in plant cells can be retraced back to an attempt to follow secretory vesicular trafficking in lily pollen tubes, which originally described this technique as evanescent wave microscopy [8]. Konopka and Bednarek further defined the method as "variableangle epifluorescence microscopy" (VAEM) to reduce background noise and to address some of the disadvantages of TIRF [9]. Mechanically, the only difference between performing microscopy in TIRF or VAEM mode is the change in orientation of the mirrors in the TIRF instrument, in which TIRF uses a single critical angle and 
VAEM ensures the use of a variety of subcritical angles. Therefore, no evanescence field but only a very thin band of illumination is generated in VAEM.

Basically, the close-up VAEM examination for cortical organelles may significantly facilitate the characterization of organelle identity, as shown in the dual-color labeling of intracellular structures in the present study. In terms of the controversy on the biological functions of ABP1, VAEM provides an alternative way to further explore the functional correlation of ABP1-tagged intracellular structures to endomembrane system. As ER acts as a gateway for the vast majority of proteins and lipids trafficking to various cellular compartments, the localization of ABP1 to ER implies potential roles of $\mathrm{ABP} 1$ at various contacting sites with other organelles. Von Wangenheim et al., reported that differential organization and motile behavior, and interactions of endosomes were related to particular root hair zones and developmental stages [45]. Interestingly, vesicular structures or endosomal compartments closely correlating with Golgi apparatuses may be actively involved in endocytic and exocytic activities in auxin signaling. Despite of the importance of local positioning, the precise connection between subcellular localization and organelle function is often not fully understood.

Vesicles are the smallest units between ER, Golgi and other endosomal compartments, and clathrin-dependent endocytosis has been extensively studies in plant cells $[16,32,46]$. Using VAEM, Konopka et al. compared dynamin-related protein 1 (DRP1) and clathrin dynamics in the cell cortex and further analyzed the functional redundancy of DRP1A and DRP1C during plant development. The results further advanced our understanding towards clathrin-mediated endocytosis and its regulation and confirming the robustness of VAEM in dissecting protein dynamics at single particle level in plant cells $[16,46]$. Recently, AtFLOT1a was implied to be involved in a clathrin-independent endocytic event, which differs dramatically in size and dynamic properties from clathrincoated vesicles [8]. Borrowing from the methodology adopted by Nakano lab in co-localizing clathrin and intracellular endosomes [32], we were able to categorize the numerous fluorescence-tagged CLC-GFP and FLOT1a puncta into different subpopulations in terms of the distance between individual puncta (Fig. 3). In the present study, the seemingly co-localized fluorescence signals of CLC-GFP and FLOT1a-mCherry under LSCM was confirmed to be not co-localized, but only partly associated indeed in the time-lapse observations with VAEM, as revealed by the results of overlapping coefficients and protein proximity index (Figs. 2 and 3). Noteworthy, overlapping coefficient has been well accepted as an index for evaluating the percentage of colocalization [32]. The sequential VAEM series can be directly imported for calculating overlapping coefficient (up to 10 sequential frames in the present study, depending on the computer memory), which is more precise and convincing for colocalization analysis in comparison to conventional LSCM.

Another advantage of VAEM is the imaging of fluorescently labeled structures closest to the coverslip $[18,44]$. Tracking cytoskeletal components under VAEM may provide a powerful tool for resolving coordinate behaviors spatio-temporally. As narrated by Staiger's lab in details, the AFs plays a central role not only in the physical organization of the cell, but also in the dynamic (re)localization of organelles, proteins, and macromolecules in response to pathogen infection [40, 47, 48]. Moreover, as tracks for the assembly and movement of defensesignaling components following pathogen perception, the AFs logically represents a virulence target for pathogens. However, a recent report shows that a type-III effector (T3E) protein from Pseudomonas syringae, HopZ1a, targets the MTs, thereby impairing the secretory pathway and suppressing cell wall-mediated defenses [49]. Similarly, AFs were stabilized through monoubiquitination of actin during infection by either pathogenic or mutualistic bacteria, but not in response to stress or viral infection [50]. This implies that the continuous rearrangements of the cytoskeleton within the same time scale may represent a surveillance mechanism to pathogens. In the present study, wild-type Col-0 plants showed significantly enhanced AF density following treatment with either flg22 or chitin, which may facilitate the endocytosis of patternrecognition receptors (PRRs) and downstream signaling in plant cells [40]. The reorientation and fragmentation of MTs in response to PAMP stimuli may be attributed to the breakdown of interaction between cytoskeletal components, as PAMP decreased the frequency of coalignment between AFs and MTs. In addition, superresolution imaging methods, such as structural illumination microscopy (SIM), have also been applied in plant cell researches, e.g. microtubular dynamics in diverse cell types and correlation of RETICULONS to primary plasmodesmata and ER, which may further expand the potential of imaging methods in tackling key biological questions [51-54].

In terms of membrane subcompartmentalization, remorins (Rem) are above all the most bona-fide marker proteins for so-termed membrane raft or microdomain in the PM, as proved by recent advancements in identification of distinct functional microdomains in living cells [21, 24]. Here, we showed that StRem1.3, AtRem1.2 and the two GPIanchored proteins were uniformly distributed on the PM in a diffused manner under LSCM, while further VAEM observations revealed relatively static punctate structures showing extremely low or only undetectable lateral motility on the PM, suggesting that microdomains 
for residing these proteins were temporally stable, as indicated by the vertical lines in the kymograph analysis. Similar results from fluorescence recovery after photobleaching (FRAP) experiments also revealed almost no photorecovery of DsRed2::AtRem 1.3 after photobleaching, indicating low mobility of these microdomainresided proteins within the PM [21]. However, another previously reported microdomain-associated protein, FLOT1a [14], displayed obvious lateral motility, which may represent active endocytic events at the sites. These results may add additional values for investigating the heterogeneity of distinct microdomains and the complexity of their coexistence.

Proteins within membranes play crucial roles in signal perception and transduction, solute partitioning, and secretion $[41,55]$. As the long-chain saturated lipid anchors found in many GPI-anchored proteins could facilitate their association with ordered-lipid microdomains or nanoclusters, we have been expecting that SKU5 and COBRA may display confined motility due to steric effects from the GPI-anchor, while integral proteins such as BOR1 may be confined to relatively limited areas. Notably, the diffusion coefficients of the proteins on the PM did not differ significantly, which were independent of the existence of membrane anchor. Therefore, GPI-anchor or other saccharide chains do not necessarily pose steric effects to influence motility parameters for protein dynamics in this case, while the properties of protein dynamics may directly correlate with their functions in specific biological context. Interestingly, Flotla displayed relatively high lateral motility in comparison to other proteins examined, which was in accordance with previous reports on their functions in constitutive or ligand-induced endocytosis $[14,19]$. It is also plausible that microdomains may act as structural units to physically separate proteins spatiotemporally and thus avoid of unexpected crosstalk in the absence of certain stimuli.

\section{Conclusion}

In summary, VAEM offers a powerful method for probing protein dynamics and other intracellular events in the proximity of the PM and the cell cortex. It may further advance our insights into membrane trafficking, cytoskeletal organization and membrane subcompartmentalization in specific contexts with improved spatial and temporal precision. With the widespread utility and increasing accessibility of VAEM, SPT and computational methodology, the future promises excitement for those interested in revealing how protein dynamics, interactions, and functions define cell-specific profiles in response to developmental cues or environmental stimuli.

\section{Additional files}

Additional file 1: Methodology for determining incident angle and

Additional file 2: Figure S1. Organellar dynamics are resolved in high spatio-temporal manner under VAEM. Figure S2. VAEM is applicable to epidermal cells of different tissues from seedlings. Figure S3. CLC-GFP tagged punctate structures correspond to clathrin-coated vesicles and Golgi apparatuses, depending on the angle of incident light. Figure S4. Actin turnover resolved using VAEM. Figure S5. Microtubular organization as revealed by VAEM. Figure S6. SKU5-GFP localizes to plasma membrane and intracellular structures. Figure S7. Distribution of the fluorescence intensity of the diffraction-limited single pCLC2- myristoyl-mGFP ${ }^{A 206 K}$ spots. Figure S8. Fluorescence correlation spectroscopy (FCS) to examine the fluorescence fluctuation in response to TyrA23 treatment. Figure S9. Analysis on MSD for different trajectories and categorization into various diffusion regimes. Figure S10. Representative kymographs for BOR1 and Lti6a. (PDF $1148 \mathrm{~kb}$ )

Additional file 3: Dynamics of Golgi apparatuses in the proximity of plasma membrane. (AVI 23044 kb)

Additional file 4: Dynamics of mitochondria in the proximity of plasma membrane. (AVI 23044 kb)

Additional file 5: Dynamics of endoplasmic reticulum (ER) tubules in the proximity of plasma membrane. (AVI $23044 \mathrm{~kb}$ )

Additional file 6: Dual-color VAEM indicates clathrin light chain (CLC)GFP and mCherry-Flot1a showing characteristic localization to separated punctate structures. (AVI $127 \mathrm{~kb}$ )

Additional file 7: Rapid turnover of actin filaments in transgenic fABD2GFP Arabidopsis seedlings in the proximity of plasma membrane. (AVI $1151 \mathrm{~kb}$ )

Additional file 8: Time-lapse recording for microtubular organization as revealed by VAEM. (AVI 1072 kb)

\section{Abbreviations}

a.u.: Arbitrary unit; ABP1: Auxin binding protein 1; AFs: Actin filaments; At: Arabidopsis thaliana; BFA: Brefeldin A; BOR1: Boron transporter 1; CLC: Clathrin light chain; DC: Diffusion coefficient; DRP1C: dynamin related protein 1C; EMCCD: Electron multiplying charge coupled device;

ER: Endoplasmic reticulum; fABD2: Filamentous-actin binding domain of fimbrin2; FCS: Fluorescence correlation spectroscopy; FLOT1a: FLOTTILIN1a; FRAP: Fluorescence recovery after photobleaching; GFP: Green fluorescent protein; GPI: Glycosyl phosphatidylinositol; HiLo microscopy: Highly inclined and laminated optical sheet microscopy; LSCM: Laser scanning confocal microscopy; LTi6a: Low temperature induced 6a; mGFP: Monomeric green fluorescent protein; mRFP: Monomeric red fluorescent protein; MSD: Mean square displacement; MTs: Microtubules; PM: Plasma membrane;

PRRs: Pattern-recognition receptors; REMs: Remorins; SIM: Structural illumination microscopy; St: Solanum tuberosum; T3E: Type-III effector; TGN: Trans-Golgi network; TIRF: Total internal reflection fluorescence; TUA5: Tubulin alpha 5; VAEM: Variable-angle epifluorescence microscopy; WCIF: Wright cell imaging facility

\section{Acknowledgements}

The authors thank Drs. Sebastian Bednarek, Niko Geldner, Thomas Ott, Akihiko Nakano, Takashi Ueda, Ines Kreuzer, Staffan Persson, John Sedbrook, and Toru Fujiwara for providing materials.

\section{Funding}

This work is supported by NSFC $(31530057,31672210,31670183)$ and Ministry of Science and Technology of China (2015BAD16B01-3). The funding bodies did not play any role in the design of the study and collection, analysis, and interpretation of data, and in writing the manuscript.

\section{Availability of data and materials}

The datasets used and / or analyzed during the current study available from the corresponding author on reasonable request. 


\section{Authors' contributions}

TC performed the experiments and wrote the manuscript; DC performed the experiments; SP supervised the research and wrote the manuscript. All authors read and approved the final manuscript.

\section{Ethics approval and consent to participate}

Not applicable

\section{Consent for publication}

Not applicable

\section{Competing interests}

The authors declare that the research was conducted in the absence of any commercial or financial relationships that could be construed as a potential conflict of interest.

\section{Publisher's Note}

Springer Nature remains neutral with regard to jurisdictional claims in published maps and institutional affiliations.

\section{Author details}

'Key Laboratory of Plant Resources, Institute of Botany, Chinese Academy of Sciences, Nanxincun 20, Xiangshan, Haidian District, Beijing 100093, China. ${ }^{2}$ University of Chinese Academy of Sciences, Beijing 100049, China. ${ }^{3}$ Key Laboratory of Post-Harvest Handling of Fruits, Ministry of Agriculture, Beijing, China.

\section{Received: 8 June 2017 Accepted: 22 January 2018}

\section{Published online: 14 March 2018}

\section{References}

1. Kusumi A, Fujiwara TK, Chadda R, Xie M, Tsunoyama TA, Kalay Z, Kasai RS, Suzuki KG. Dynamic organizing principles of the plasma membrane that regulate signal transduction: commemorating the fortieth anniversary of singer and Nicolson's fluid-mosaic model. Annu Rev Cell Dev Biol. 2012;28:215-50.

2. Malinsky J, Opekarova M, Grossmann G, Tanner W. Membrane microdomains, rafts, and detergent-resistant membranes in plants and fungi. Annu Rev Plant Biol. 2013;64:501-29.

3. Gell C, Berndt M, Enderlein J, Diez STIRF. Microscopy evanescent field calibration using tilted fluorescent microtubules. J Microsc. 2009;234:38-46.

4. Li X, Luu DT, Maurel C, Lin J. Probing plasma membrane dynamics at the single-molecule level. Trends Plant Sci. 2013;18:617-24.

5. Leonard D, Hayakawa A, Lawe D, Lambright D, Bellve KD, Standley C, Lifshitz LM, Fogarty KE, Corvera S. Sorting of EGF and transferrin at the plasma membrane and by cargo-specific signaling to EEA1-enriched endosomes. J Cell Sci. 2008;121:3445-58.

6. Webb RL, Rozov O, Watkins SC, McCartney BM. Using total internal reflection fluorescence (TIRF) microscopy to visualize cortical actin and microtubules in the drosophila syncytial embryo. Dev Dyn. 2009;238:2622-32.

7. Smith IF, Parker I. Imaging the quantal substructure of single IP3R channel activity during $\mathrm{Ca}^{2+}$ puffs in intact mammalian cells. Proc Natl Acad Sci U S A. 2009;106:6404-9.

8. Wang X, Teng Y, Wang Q, Li X, Sheng X, Zheng M, Samaj J, Baluska F, Lin J. Imaging of dynamic secretory vesicles in living pollen tubes of Picea meyeri using evanescent wave microscopy. Plant Physiol. 2006:141:1591-603.

9. Konopka CA, Bednarek SY. Variable-angle epifluorescence microscopy: a new way to look at protein dynamics in the plant cell cortex. Plant J. 2008; 53(1):186-96.

10. Wan Y, Ash WM 3rd, Fan L, Hao H, Kim MK, Lin J. Variable-angle total internal reflection fluorescence microscopy of intact cells of Arabidopsis thaliana. Plant Methods. 2011;7:27.

11. Tokunaga M, Imamoto N, Sakata-Sogawa K. Highly inclined thin illumination enables clear single-molecule imaging in cells. Nat Methods. 2008:5:159-61.

12. Clough SJ, Bent AF. Floral dip: a simplified method for agrobacteriummediated transformation of Arabidopsis thaliana. Plant J. 1998;16:735-43.

13. Murashige T, Skoog FA. Revised medium for rapid growth and bio assays with tobacco tissue cultures. Physiol Plant. 1962;15:473-97.

14. Li R, Liu P, Wan Y, Chen T, Wang Q, Mettbach U, Baluska F, Samaj J, Fang X, Lucas WJ, et al. A membrane microdomain-associated protein, Arabidopsis Flot1, is involved in a clathrin-independent endocytic pathway and is required for seedling development. Plant Cell. 2012;24:2105-22.
15. Dhonukshe P, Aniento F, Hwang I, Robinson DG, Mravec J, Stierhof YD, Friml J. Clathrin-mediated constitutive endocytosis of PIN auxin efflux carriers in Arabidopsis. Curr Biol. 2007;17:520-7.

16. Konopka CA, Backues SK, Bednarek SY. Dynamics of Arabidopsis dynaminrelated protein $1 \mathrm{C}$ and a clathrin light chain at the plasma membrane. Plant Cell. 2008;20:1363-80.

17. Cutler SR, Ehrhardt DW, Griffitts JS, Somerville CR, Random GFP. CDNA fusions enable visualization of subcellular structures in cells of Arabidopsis at a high frequency. Proc Natl Acad Sci U S A. 2000;97:3718-23.

18. Sampathkumar A, Lindeboom JJ, Debolt S, Gutierrez R, Ehrhardt DW, Ketelaar T, Persson S. Live cell imaging reveals structural associations between the actin and microtubule cytoskeleton in Arabidopsis. Plant Cell. 2011:23:2302-13.

19. Takano J, Tanaka M, Toyoda A, Miwa K, Kasai K, Fuji K, Onouchi H, Naito S, Fujiwara T. Polar localization and degradation of Arabidopsis boron transporters through distinct trafficking pathways. Proc Natl Acad Sci U S A. 2010;107:5220-5.

20. Sedbrook JC, Carroll KL, Hung KF, Masson PH, Somerville CR. The Arabidopsis SKU5 gene encodes an extracellular glycosyl phosphatidylinositol-anchored glycoprotein involved in directional root growth. Plant Cell. 2002;14:1635-48.

21. Demir F, Horntrich C, Blachutzik JO, Scherzer S, Reinders Y, Kierszniowska S, Schulze WX, Harms GS, Hedrich R, Geiger D, et al. Arabidopsis nanodomaindelimited ABA signaling pathway regulates the anion channel SLAH3. Proc Natl Acad Sci U S A. 2013;110:8296-301.

22. Roudier F, Fernandez AG, Fujita M, Himmelspach R, Borner GH, Schindelman G, Song S, Baskin TI, Dupree P, Wasteneys GO, et al. COBRA, an Arabidopsis extracellular glycosyl-phosphatidyl inositol-anchored protein, specifically controls highly anisotropic expansion through its involvement in cellulose microfibril orientation. Plant Cell. 2005:17:1749-63.

23. Hemsley PA, Weimar T, Lilley KS, Dupree P, Grierson CSA. Proteomic approach identifies many novel palmitoylated proteins in Arabidopsis. New Phytol. 2013;197:805-14.

24. Jarsch IK, Konrad SS, Stratil TF, Urbanus SL, Szymanski W, Braun P, Braun KH, Ott T. Plasma membranes are subcompartmentalized into a plethora of coexisting and diverse microdomains in Arabidopsis and Nicotiana benthamiana. Plant Cell. 2014;26:1698-711.

25. Li Q, Lau A, Morris TJ, Guo L, Fordyce CB, Stanley EF. A syntaxin 1, Galpha(o), and $\mathrm{N}$-type calcium channel complex at a presynaptic nerve terminal: analysis by quantitative immunocolocalization. J Neurosci. 2004;24:4070-81.

26. Higaki T, Kutsuna N, Sano T, Kondo N, Hasezawa S. Quantification and cluster analysis of actin cytoskeletal structures in plant cells: role of actin bundling in stomatal movement during diurnal cycles in Arabidopsis guard cells. Plant J. 2010;61:156-65.

27. Li J, Henty-Ridilla JL, Staiger BH, Day B, Staiger CJ. Capping protein integrates multiple MAMP signalling pathways to modulate actin dynamics during plant innate immunity. Nat Commun. 2015:6:7206.

28. Jaqaman K, Loerke D, Mettlen M, Kuwata H, Grinstein S, Schmid SL, Danuser G. Robust single-particle tracking in live-cell time-lapse sequences. Nat Methods. 2008;5:695-702.

29. Wieser S, Schutz GJ. Tracking single molecules in the live cell plasma membrane-Do's and Don't's. Methods. 2008:46:131-40.

30. Goulian M, Simon SM. Tracking single proteins within cells. Biophys J. 2000; 79:2188-98

31. Saxton MJ. Single-particle tracking: the distribution of diffusion coefficients. Biophys J. 1997;72:1744-53.

32. Ito E, Fujimoto $M$, Ebine $K$, Uemura T, Ueda T, Nakano A. Dynamic behavior of clathrin in Arabidopsis Thaliana unveiled by live imaging. Plant J. 2012;69:204-16.

33. Zinchuk V, Wu Y, Grossenbacher-Zinchuk O, Stefani E. Quantifying spatial correlations of fluorescent markers using enhanced background reduction with protein proximity index and correlation coefficient estimations. Nat Protoc. 2011:6:1554-67.

34. Gao $Y$, Zhang $Y$, Zhang D, Dai $X$, Estelle M, Zhao $Y$. Auxin binding protein 1 (ABP1) is not required for either auxin signaling or Arabidopsis development. Proc Natl Acad Sci U S A. 2015;112:2275-80.

35. Grebe M, Xu J, Mobius W, Ueda T, Nakano A, Geuze HJ, Rook MB, Scheres B. Arabidopsis sterol endocytosis involves actin-mediated trafficking via ARA6positive early endosomes. Curr Biol. 2003:13:1378-87.

36. Logan DC, Leaver CJ. Mitochondria-targeted GFP highlights the heterogeneity of mitochondrial shape, size and movement within living plant cells. J Exp Bot. 2000;51:865-71. 
37. Fan $L$, Hao H, Xue $Y$, Zhang L, Song K, Ding Z, Botella MA, Wang H, Lin J. Dynamic analysis of Arabidopsis AP2 sigma subunit reveals a key role in clathrin-mediated endocytosis and plant development. Development. 2013; 140:3826-37.

38. Ritzenthaler C, Nebenfuhr A, Movafeghi A, Stussi-Garaud C, Behnia L, Pimpl $P$, Staehelin LA, Robinson DG. Reevaluation of the effects of brefeldin a on plant cells using tobacco bright yellow 2 cells expressing Golgi-targeted green fluorescent protein and COPI antisera. Plant Cell. 2002;14:237-61.

39. Staiger CJ, Sheahan MB, Khurana P, Wang X, McCurdy DW, Blanchoin L. Actin filament dynamics are dominated by rapid growth and severing activity in the Arabidopsis cortical array. J Cell Biol. 2009;184:269-80.

40. Henty-Ridilla JL, Li J, Day B, Staiger CJ. ACTIN DEPOLYMERIZING FACTOR4 regulates actin dynamics during innate immune signaling in Arabidopsis. Plant Cell. 2014;26:340-52.

41. Keinath NF, Kierszniowska S, Lorek J, Bourdais G, Kessler SA, ShimosatoAsano H, Grossniklaus U, Schulze WX, Robatzek S, Panstruga R. PAMP (pathogen-associated molecular pattern)-induced changes in plasma membrane compartmentalization reveal novel components of plant immunity. J Biol Chem. 2010;285:39140-9.

42. Schindelman G, Morikami A, Jung J, Baskin TI, Carpita NC, Derbyshire P, McCann MC, Benfey PN. COBRA encodes a putative GPI-anchored protein, which is polarly localized and necessary for oriented cell expansion in Arabidopsis. Genes Dev. 2001;15:1115-27.

43. Schwacke R, Schneider A, van der Graaff E, Fischer K, Catoni E, Desimone M, Frommer WB, Flugge UI, Kunze R. ARAMEMNON, a novel database for Arabidopsis integral membrane proteins. Plant Physiol. 2003;131:16-26.

44. Qu X, Zhang H, Xie Y, Wang J, Chen N, Huang S. Arabidopsis villins promote actin turnover at pollen tube tips and facilitate the construction of actin collars. Plant Cell. 2013;25:1803-17.

45. von Wangenheim D, Rosero A, Komis G, Samajova O, Ovecka M, Voigt B, Samaj J. Endosomal interactions during root hair growth. Front Plant Sci. 2015;6:1262. https://doi.org/10.3389/fpls.2015.01262.

46. Konopka CA, Bednarek SY. Comparison of the dynamics and functional redundancy of the Arabidopsis dynamin-related isoforms DRP1A and DRP1C during plant development. Plant Physiol. 2008;147:1590-602.

47. Day B, Henty JL, Porter KJ, Staiger CJ. The pathogen-actin connection: a platform for defense signaling in plants. Annu Rev Phytopathol. 2011;49: 483-506.

48. Henty-Ridilla JL, Shimono M, Li J, Chang JH, Day B, Staiger CJ. The plant actin cytoskeleton responds to signals from microbe-associated molecular patterns. PLoS Pathog. 2013;9:e1003290.

49. Lee AH, Hurley B, Felsensteiner C, Yea C, Ckurshumova W, Bartetzko V, Wang PW, Quach V, Lewis JD, Liu YC, et al. A bacterial acetyltransferase destroys plant microtubule networks and blocks secretion. PLoS Pathog. 2012;8:e1002523.

50. Dantan-Gonzalez E, Rosenstein Y, Quinto C, Sanchez F. Actin monoubiquitylation is induced in plants in response to pathogens and symbionts. Mol Plant-Microbe Interact. 2001;14:1267-73.

51. Knox K, Wang P, Kriechbaumer V, Tilsner J, Frigerio L, Sparkes I, Hawes C, Oparka K. Putting the squeeze on plasmodesmata: a role for reticulons in primary plasmodesmata formation. Plant Physiol. 2015;168:1563-72.

52. Komis G, Mistrik M, Samajova O, Doskocilova A, Ovecka M, Illes P, Bartek J, Dynamics SJ. Organization of cortical microtubules as revealed by superresolution structured illumination microscopy. Plant Physiol. 2014;165:129-48.

53. Komis G, Mistrik M, Samajova O, Ovecka M, Bartek J, Samaj J. Superresolution live imaging of plant cells using structured illumination microscopy. Nat Protoc. 2015;10:1248-63.

54. Komis G, Samajova O, Ovecka M, Samaj J. Super-resolution microscopy in plant cell imaging. Trends Plant Sci. 2015;20:834-43.

55. Robert S, Kleine-Vehn J, Barbez E, Sauer M, Paciorek T, Baster P, Vanneste S, Zhang J, Simon S, Covanova M, et al. ABP1 mediates auxin inhibition of clathrin-dependent endocytosis in Arabidopsis. Cell. 2010;143:111-21.

\section{Submit your next manuscript to BioMed Central and we will help you at every step:}

- We accept pre-submission inquiries

- Our selector tool helps you to find the most relevant journal

- We provide round the clock customer support

- Convenient online submission

- Thorough peer review

- Inclusion in PubMed and all major indexing services

- Maximum visibility for your research

Submit your manuscript at www.biomedcentral.com/submit
Biomed Central 